\title{
Charge and Magnetization Inhomogeneities in Diluted Magnetic Semiconductors
}

\begin{abstract}
Carsten Timm*
Institut für Theoretische Physik, Freie Universität Berlin, Arnimallee 14, D-14195 Berlin, Germany, and Department of Physics and Astronomy, University of Kansas, Lawrence, Kansas 66045, USA

(Received 3 October 2005; revised manuscript received 22 December 2005; published 20 March 2006; corrected 19 April 2006)

It is predicted that III-V diluted magnetic semiconductors can exhibit stripelike modulations of magnetization and carrier concentration. This inhomogeneity results from the strong dependence of the magnetization on the carrier concentration. Within Landau theory, a characteristic temperature $T^{*}$ below the Curie temperature is found so that below $T^{*}$ the equilibrium magnetization shows modulations, which are strongly anharmonic. The wavelength and amplitude of the modulation rise for decreasing temperature, starting from zero at $T^{*}$. Above $T^{*}$, the equilibrium state is homogeneous, but the coupling between charge and magnetization leads to the appearance of an electrically charged layer in domain walls.
\end{abstract}

DOI: 10.1103/PhysRevLett.96.117201

Introduction. - Diluted magnetic semiconductors (DMS) are investigated extensively as promising materials for spintronics applications [1,2] and because of their unique physical properties $[3,4]$. Since the magnetic interaction is mediated by the carriers, the magnetization and the Curie temperature increase for increasing carrier concentration [5-10]. In fact, the magnetization can be changed in situ by tuning the carrier concentration with a gate voltage $[5,6,8]$.

Coupling between magnetism and carrier concentration can lead to inhomogeneous equilibrium states, as found in manganites [11], nickelates [12], and cuprates [13]. The present Letter analyzes this possibility for III-V DMS, concentrating on stripelike, one-dimensional variations of the magnetizations. We employ a Landau theory for the coupled magnetic and charge degrees of freedom. This approach is valid on length scales on which the impurity distribution can be treated as homogeneous [14]. The characteristic length scale is $n_{\mathrm{Mn}}^{-1 / 3}$, where $n_{\mathrm{Mn}}$ is the density of Mn impurities [4,15]. Finally, we discuss possible experiments.

Magnetic domains have also been observed in (Ga, Mn)As [16,17]. They are formed to reduce the dipolar energy, as in other ferromagnets. One can expect the coupling between magnetization and carriers to lead to an inhomogeneous charge profile in a domain wall. This question is addressed in the final part of this Letter.

Landau theory. - We write the Hamiltonian as a functional of magnetization $\mathbf{m}$ and deviation of carrier density from its spatial average $\delta n \equiv n-\bar{n}$. Charge neutrality requires $\int d^{3} r \delta n=0$. The magnetic part has the usual form

$$
H_{m}=\int d^{3} r\left\{\frac{\alpha}{2} m^{2}+\frac{\beta}{4} m^{4}+\frac{\gamma}{2} \partial_{i} \mathbf{m} \cdot \partial_{i} \mathbf{m}\right\},
$$

where $\partial_{i} \equiv \partial / \partial r_{i}$ and summation over $i$ is implied. The mean-field Curie temperature is determined by $\alpha=0$. Since experimentally the Curie temperature depends approximately linearly on carrier concentration, we expand
PACS numbers: 75.50.Pp, 75.10.Hk, 75.30.Fv, 75.60.Ch

$\alpha=\alpha^{\prime}\left(T-T_{c}-\eta \delta n\right)$, where $T_{c}$ is the Curie temperature for $\delta n=0$. This dependence of $\alpha$ provides the coupling between magnetism and carrier concentration in our model and is responsible for the physics discussed in the following. Since the equilibrium magnetization for constant $\alpha$ is $m_{0} \equiv \sqrt{-\alpha / \beta}$, larger magnetization is favored in regions with higher carrier concentration (note that $\alpha<$ 0 in the ferromagnetic phase).

The second ingredient for our model is the screened Coulomb energy due to the charge inhomogeneity,

$$
H_{\delta n}=\frac{1}{2} \int d^{3} r d^{3} r^{\prime} \frac{e^{2}}{4 \pi \epsilon_{0} \epsilon} \delta n(\mathbf{r}) \delta n\left(\mathbf{r}^{\prime}\right) \frac{e^{-\left|\mathbf{r}-\mathbf{r}^{\prime}\right| / r_{0}}}{\left|\mathbf{r}-\mathbf{r}^{\prime}\right|} .
$$

The total Hamiltonian is $H=H_{m}+H_{\delta n}$.

We first discuss qualitatively what kind of equilibrium states we expect from $H$. Any inhomogeneous charge distribution increases $H_{\delta n}$. On the other hand, the contribution from $H_{m}$ is not obvious, since the first term is negative for $T<T_{c}+\eta \delta n$. We will see that the magnetic energy decrease in regions of higher carrier concentration and magnetization can outweigh the increase in regions of lower $\delta n$ and $m$ and even the increase in electrostatic energy. In that case, the equilibrium state is indeed inhomogeneous. We consider stripelike, one-dimensional modulations. Two- and three-dimensional patterns seem less likely, because they contain more regions with large magnetization gradients for a given inhomogeneity length scale, which increase the energy due to the gradient term in $H_{m}$. One could expect the inhomogeneity to take the form of stripe domains [16] with alternating magnetization. However, we will see that the equilibrium solution shows a magnetization modulation without sign change.

We now turn to the formal derivation of equilibrium states. It is convenient to express $H_{\delta n}$ in terms of the electrostatic potential $\phi$. With $\left(\Delta-r_{0}^{-2}\right) \phi(\mathbf{r})=-e \delta n(\mathbf{r}) / \epsilon_{0} \epsilon$ (for $p$-type DMS), we obtain the total Hamiltonian

$$
H=\int d^{3} r\left\{\frac{\alpha^{\prime}\left(T-T_{c}\right)}{2} m^{2}+\frac{\beta}{4} m^{4}+\frac{\gamma}{2} \partial_{i} \mathbf{m} \cdot \partial_{i} \mathbf{m}+\frac{\epsilon_{0} \epsilon}{2 r_{0}^{2}} \phi^{2}+\frac{\epsilon_{0} \epsilon}{2} \partial_{i} \phi \partial_{i} \phi-\frac{\alpha^{\prime} \eta \epsilon_{0} \epsilon}{2 e r_{0}^{2}} m^{2} \phi-\frac{\alpha^{\prime} \eta \epsilon_{0} \epsilon}{e} \mathbf{m} \cdot\left(\partial_{i} \mathbf{m}\right) \partial_{i} \phi\right\} .
$$


Equilibrium configurations are given by minima of $H$ subject to the constraint of charge neutrality $\int d^{3} r(\Delta-$ $\left.r_{0}^{-2}\right) \phi=0$, which is implemented with a Lagrange multiplier. Introducing the averaged squared magnetization $\overline{\mathrm{m}^{2}}$ and the rescaled potential $\Phi=\left(\alpha^{\prime} \eta \epsilon_{0} \epsilon / e\right) \phi$, we obtain two coupled Euler equations for $\mathbf{m}$ and $\Phi$. Eliminating $\Phi$ from the first, we find

$$
\begin{aligned}
0= & -a \mathbf{m} \partial_{i}\left(\mathbf{m} \cdot \partial_{i} \mathbf{m}\right)+b \Delta \mathbf{m} \\
& -\underbrace{\left(c+\frac{a}{2 r_{0}^{2}} \overline{m^{2}}\right)}_{c^{\prime}} \mathbf{m}-\underbrace{\left(d-\frac{a}{2 r_{0}^{2}}\right)}_{d^{\prime}} m^{2} \mathbf{m}
\end{aligned}
$$

and

$$
\left(\Delta-r_{0}^{-2}\right) \Phi=\frac{a}{2}\left(\Delta-r_{0}^{-2}\right)\left(m^{2}-\overline{m^{2}}\right),
$$

with $a \equiv \alpha^{\prime 2} \eta^{2} \epsilon_{0} \epsilon / e^{2}, b \equiv \gamma, c \equiv \alpha^{\prime}\left(T-T_{c}\right), d \equiv \beta$. Equation (4) retains information about the coupling to the carrier density since $a \propto \eta^{2}$. The only bounded solution of Eq. (5) is $\Phi=(a / 2)\left(m^{2}-\overline{m^{2}}\right)$. The equations support the homogeneous mean-field solution $m^{2}=m_{0}^{2} \equiv$ $-c / d>0, \Phi=0$ for $T<T_{c}$. While Eqs. (4) and (5) contain five parameters, it is sufficient to vary only two to obtain all possible solutions up to rescaling. We choose $a / d r_{0}^{2} \propto \eta^{2}$ and $c r_{0}^{2} / b \propto T-T_{c}$.

Periodic solutions. - For periodic, collinear solutions that depend only on $x$, Eq. (4) becomes

$$
0=-a m \partial_{x}\left(m \partial_{x} m\right)+b \partial_{x}^{2} m-c^{\prime} m-d^{\prime} m^{3} .
$$

This is an integro-differential equation due to the term $\overline{m^{2}}=(1 / \lambda) \int_{0}^{\lambda} d x m^{2}(x)$ in $c^{\prime}$, where $\lambda$ is the wavelength. $c^{\prime}$ is determined self-consistently below. Boundary conditions $m(0)=m^{*}$ and $\partial_{x} m(0)=0$ are imposed, where $m^{*}$ will be obtained by minimizing the energy.

Using standard methods, we obtain the explicit integral for the inverse function on the interval $[-\lambda / 2, \lambda / 2]$,

$$
x= \pm \int_{m^{*}}^{m} d \tilde{m} \sqrt{\frac{b-a \tilde{m}^{2}}{c^{\prime} \tilde{m}^{2}+d^{\prime} \tilde{m}^{4} / 2-c^{\prime} m^{* 2}-d^{\prime} m^{* 4} / 2}} .
$$

This expression satisfies $\partial_{x} m(0)=0$, since $\partial x / \partial m$ diverges at $m=m^{*}$. To obtain a periodic function, the denominator must have another zero at the next extremum of $m(x)$ at $x= \pm \lambda / 2$. Beyond $\pm \lambda / 2$, the solution continues periodically.

A special role is played by the magnetization value $m_{\text {sing }} \equiv \sqrt{b / a}$ : Here $\partial x / \partial m$ vanishes so that coordinates $x$ beyond this point normally cannot be reached and there is no solution for all $x$. However, a solution (here called type 1) crossing $m=m_{\text {sing }}$ is possible if numerator and denominator vanish simultaneously. This solution is $m(x)=\left(2 m_{\min }^{2}-m_{\text {sing }}^{2}\right)^{1 / 2} \cos \left(\sqrt{d^{\prime} / 2 a} x\right)$, where $m_{\min } \equiv$ $\sqrt{-c^{\prime} / d^{\prime}}$ is the minimum of the denominator.

For all other periodic solutions, $m$ must be either larger or smaller than $m_{\text {sing }}$ everywhere. For $m^{*}<m_{\text {sing }}$, periodic solutions (type 2) oscillating between $m^{*}$ and $-m^{*}$ with zero average exist if $m^{*}<m_{\min }$. For $m^{*}>m_{\text {sing }}$, periodic solutions (type 3) oscillating around $m_{\min }$ exist for $c^{\prime} m^{* 2}+$ $d^{\prime} m^{* 4} / 2<c^{\prime} m_{\text {sing }}^{2}+d^{\prime} m_{\text {sing }}^{4} / 2$; see Fig. 1 .

Next the phase diagram is mapped back onto the parameters of the Euler equation (6), determining $\overline{m^{2}}$ selfconsistently. The results are shown in Fig. 2(a) for $a / d r_{0}^{2}=$ 1. The diagram for other values has the same topology. Figure 2(b) shows the resulting phase diagram in terms of $c r_{0}^{2} / b$ and general $d r_{0}^{2} / a \propto m_{\text {sing. }}^{2}$. The lines A, B, and C show the shift of the crossing points marked A, B, and C, respectively, in Fig. 2(a) with $d r_{0}^{2} / a$. Type 3 solutions exist to the left of point A, i.e., for $m_{0}>m_{\text {sing }}$.

From Eqs. (3)-(5), we find the average energy density

$$
\bar{e}=\frac{1}{\lambda} \int_{0}^{\lambda} d x\left\{-\frac{d^{\prime}}{4} m^{4}+\frac{a}{2} m^{2}\left(\partial_{x} m\right)^{2}\right\}-\frac{a}{8 r_{0}^{2}}\left(\overline{m^{2}}\right)^{2} .
$$

For the homogeneous solution, we obtain the standard result $\bar{e}=e_{\text {hom }} \equiv-c^{2} / 4 d$. Numerical evaluation shows that type 1 and 2 (3) solutions always have higher (lower) energy than the homogeneous solution. Among type 3 solutions, the energy is minimized by the maximum amplitude, where $m$ comes arbitrarily close to $m_{\text {sing }}$.

The mean-field magnetization of our DMS model is thus zero for $T \geq T_{c}$, homogeneous for $T^{*} \leq T<T_{c}$, where

$$
T^{*} \equiv T_{c}-\frac{e^{2}}{\epsilon_{0} \epsilon} \frac{\beta \gamma}{\alpha^{13} \eta^{2}}
$$

corresponds to line A in Fig. 2(b), and a periodic spindensity and charge-density wave for $T<T^{*}$. The dipolar interaction omitted here favors $\mathbf{m}$ lying in the $y z$ plane. The magnetization and potential show sharp cusps at the min-

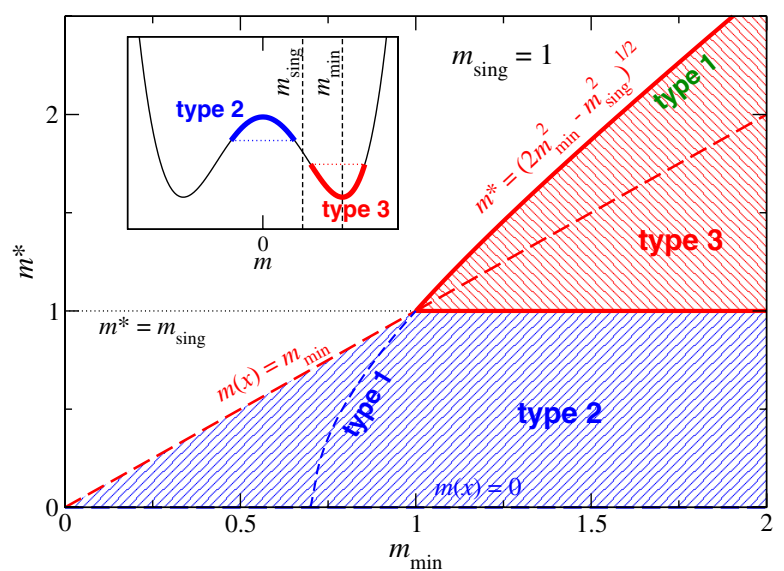

FIG. 1 (color online). Phase diagram for periodic solutions for the magnetization in terms of $m_{\min }$ and $m^{*}=m(0)$ for $m_{\text {sing }}=1$. Type 1 solutions exist for $m^{*}=\left(2 m_{\min }^{2}-m_{\text {sing }}^{2}\right)^{1 / 2}$ and become a special case of type 2 for $m^{*}<m_{\text {sing }}$. Type 2 solutions exist for $m^{*}<\min \left(m_{\min }, m_{\text {sing }}\right)$ and type 3 solutions for $m_{\text {sing }}<m^{*}<$ $\left(2 m_{\text {min }}^{2}-m_{\text {sing }}^{2}\right)^{1 / 2}$. The homogeneous solutions are also shown. Inset: Schematic plot of the denominator in Eq. (7) showing the values assumed by $m$ for type 2 and 3 solutions. 


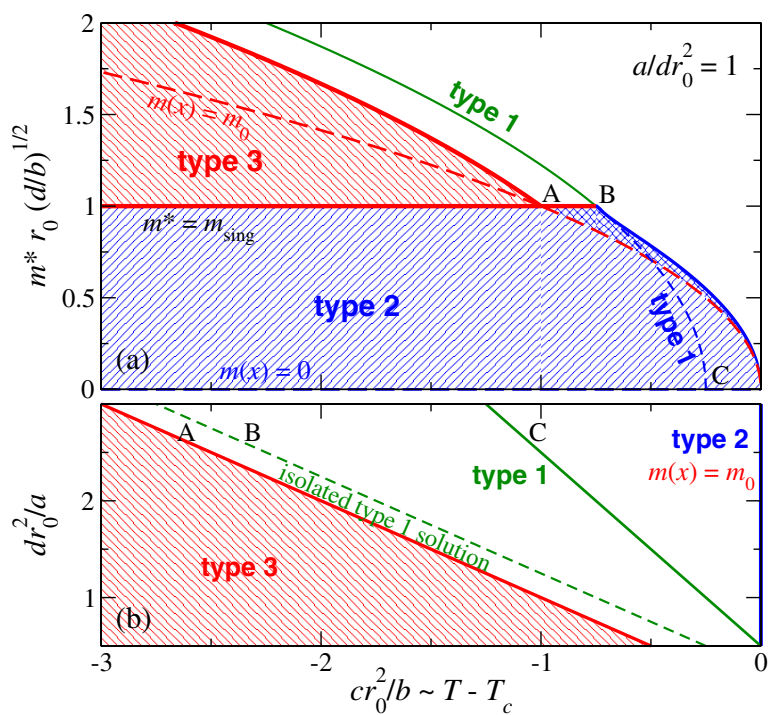

FIG. 2 (color online). (a) Phase diagram for periodic solutions for the magnetization in terms of $c r_{0}^{2} / b \propto T-T_{c}$ and initial magnetization $m^{*}=m(0)$ for $a / d r_{0}^{2}=1$. The symbols are as in Fig. 1. In the distorted triangle with corners A, B, 0, two solutions with different wavelength coexist. (b) Phase diagram for periodic magnetization solutions in terms of $c r_{0}^{2} / b \propto T-T_{c}$ and $d r_{0}^{2} / a \propto m_{\text {sing }}^{2}$. The various solutions exist to the left of the respective lines.

ima of $m$; see the inset in Fig. 3. The cusps lead to negative peaks in the carrier density, which become $\delta$ functions for $m^{*} \rightarrow m_{\text {sing. }}$. This divergence is cut off by the condition of non-negative hole concentration. Since the amplitude, wavelength, and energy approach finite values for $m^{*} \rightarrow$ $m_{\text {sing }}$, the Landau theory gives a good impression of the profile, except for some broadening of the cusps.

The optimum solution can be written down explicitly:

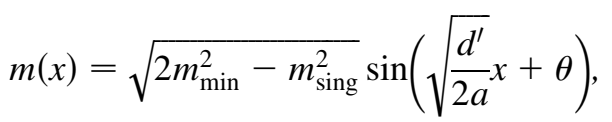

for $0 \leq x \leq \lambda$ and periodically repeated. Here $\left(2 m_{\min }^{2}-\right.$ $\left.m_{\text {sing }}^{2}\right)^{1 / 2} \sin \theta=m_{\text {sing. }}$. From $m(x)$, one can obtain expressions for the wavelength $\lambda=2\left(2 a / d^{\prime}\right)^{1 / 2}(\pi / 2-\theta)$, the average magnetization $\bar{m}$, the peak-to-peak amplitude $\delta m_{\mathrm{pp}}=\left(2 m_{\text {min }}^{2}-m_{\text {sing }}^{2}\right)^{1 / 2}-m_{\text {sing }}$, and the energy; see Fig. 3. Note that $\bar{m}$ is nonzero for all $T<T_{c}$. Close to $T^{*}$, the wavelength becomes small. In this regime, the continuum theory breaks down, since $\lambda$ is not large compared to the disorder length scale. Figure 3 also shows that the fundamental length scale is the screening length $r_{0}$. The inset shows a typical solution.

It is important to check whether the periodic solution can occur in real DMS. For that, $T_{c}-T^{*}$ should be small. Equation (9) shows that this is the case for high dielectric constant, small spin stiffness, strong dependence of $T_{c}$ on carrier concentration, and rapid onset of magnetization below $T_{c}$. For $(\mathrm{Ga}, \mathrm{Mn}) \mathrm{As}$, we estimate $T^{*}$ by comparing experiments $[7,9]$ to mean-field theory for homogeneous

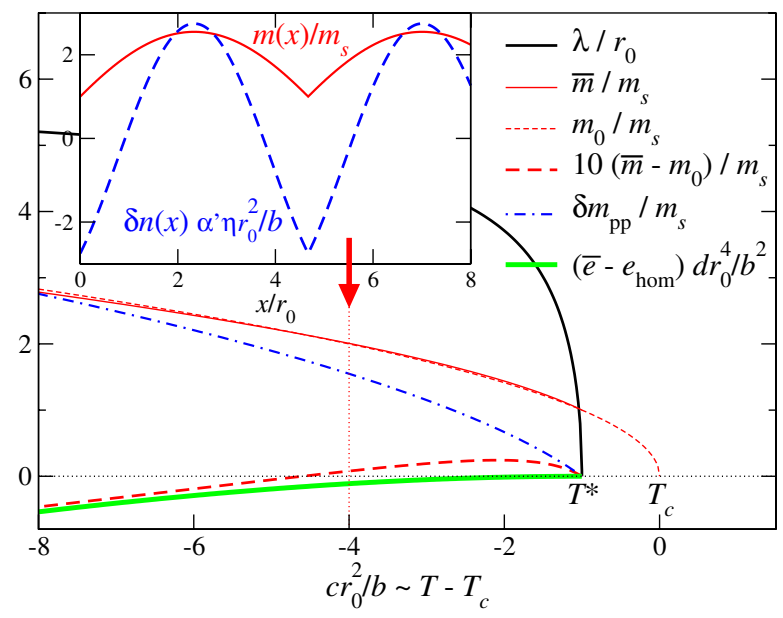

FIG. 3 (color online). Wave length $\lambda$, average magnetization $\bar{m}$, peak-to-peak amplitude $\delta m_{\mathrm{pp}}$, and gain in energy density $\bar{e}-$ $e_{\text {hom }}$ for the periodic magnetization with lowest energy. The magnetization for the homogeneous solution is also shown. The unit of magnetization is $m_{s} \equiv \sqrt{b / d} / r_{0} . a / d r_{0}^{2}=1$ is assumed. Inset: Magnetization and excess carrier density for $c r_{0}^{2} / b=-4$.

magnetization [14] and to spin-wave theory [18]. We find $T_{c}-T^{*}$ of the order of $10 \mathrm{~K}$. The properties of $(\mathrm{Ga}, \mathrm{Mn}) \mathrm{As}$ vary strongly with $\mathrm{Mn}$ concentration and growth procedures. In particular, $T_{c}-T^{*}$ is inversely proportional to the square of the shift of $T_{c}$ with carrier concentration $\eta^{2}$. In Ref. [9], $\eta \sim 5.4 \times 10^{5} \mathrm{~K}^{3}$, which was used for the above estimate, whereas in Ref. [7], $\eta \sim 1.5 \times 10^{5} \mathrm{~K} \AA^{3}$, which would increase $T_{c}-T^{*}$.

Figure 3 suggests that measurements of the average magnetization, which have been performed extensively, are unlikely to find evidence for the inhomogeneous state. For that, probes sensitive to the spatial variation are required. For example, the magnetic modulation should be observable in neutron-scattering experiments. In real space, magnetic scanning-tunneling microscopy (STM) and, for large $\lambda$, scanning Hall probe experiments [16] or magneto-optical techniques [17] are promising. Conversely, the modulation in carrier concentration should be observable in optical reflection or transmission for large enough $\lambda$. It also leads to a modulation of the local density of states which could be probed by STM. The smoking gun experiment would be to look for charge and magnetization modulations of the same wavelength.

Domain walls. -Finally, we study the effect of magnetization-carrier coupling on domain walls $[16,17]$. We restrict ourselves to solutions that are homogeneous in the $y, z$ directions. Equations (4) and (5) are solved under the boundary conditions $\lim _{x \rightarrow \pm \infty} \mathbf{m}(x)= \pm m_{0} \hat{\mathbf{z}}$, where $\hat{\mathbf{z}}$ is the unit vector in the $z$ direction. Since $m^{2}(x)$ only deviates appreciably from $m_{0}^{2}$ in a finite interval, we have $\overline{m^{2}}=m_{0}^{2}=-c / d$ in the limit of infinite system size $L \rightarrow$ $\infty$. However, it turns out that charge neutrality can be satisfied only by keeping terms of order $1 / L$ in $\overline{m^{2}}$. One such term comes from the region far from the wall, where 


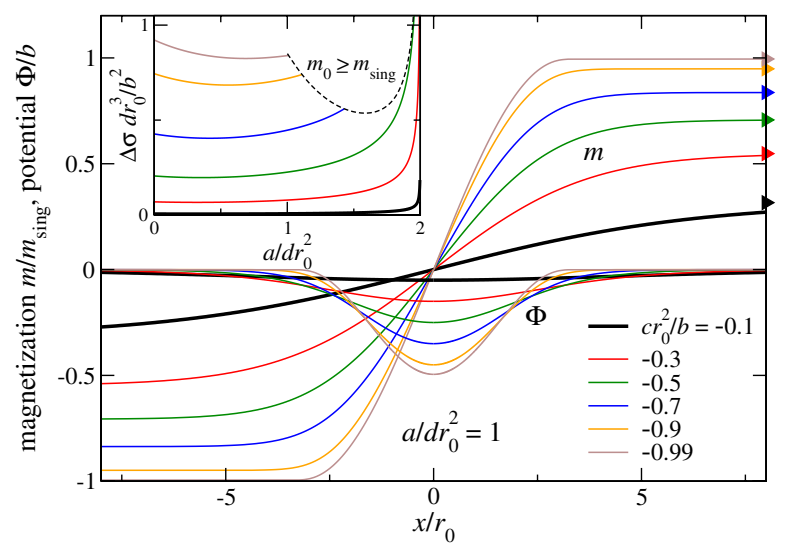

FIG. 4 (color online). Magnetization and electrostatic potential for typical domain-wall solutions for $a / d r_{0}^{2}=1$. The curves are for $c r_{0}^{2} / b=-0.1,-0.3,-0.5,-0.7,-0.9,-0.99$. The asymptotical values $m_{0}=m(x \rightarrow \infty)$ are indicated by triangles. Inset: Energy density $\Delta \sigma$ of a domain wall as a function of coupling strength for the same values of $c$.

we write $m(L / 2) \cong m_{0}+m_{1} / L$. This means that the enhanced carrier density compensating the reduction in the wall is spread out over the bulk.

With the additional condition $m(0)=0$, we obtain

$$
x=\int_{0}^{m} d \tilde{m} \sqrt{\frac{b-a \tilde{m}^{2}}{c^{\prime} \tilde{m}^{2}+d^{\prime} \tilde{m}^{4} / 2-c^{\prime} m_{0}^{2}-d^{\prime} m_{0}^{4} / 2}},
$$

with $c^{\prime}=c-\left(a / 2 r_{0}^{2}\right)(c / d)$ and $d^{\prime}=d-a / 2 r_{0}^{2}$. The integrand must be free of singularities for $0 \leq \tilde{m}<m_{0}$, which implies $m_{0} \leq m_{\text {sing. }}$. Thus, domain-wall solutions exist only for $T^{*} \leq T<T_{c}$ [19]; see Fig. 2(a). This indicates that $T_{c}-T^{*}$ is large for samples which show domains at low temperatures.

Equation (11) can be integrated explicitly. It also yields an expression for the typical width $\xi_{w} \equiv m_{0} / \partial_{x} m(0)$ of a domain wall, $\xi_{w}^{2}=-2 b d / c\left(d-a / 2 r_{0}^{2}\right) . \xi_{w}$ increases for increasing coupling $a \propto \eta^{2}$ between magnetism and carriers due to their Coulomb repulsion. Figure 4 shows $m(x)$ and $\Phi(x)$ for typical domain-wall solutions. The excess carrier concentration $\delta n \propto\left(r_{0}^{-2}-\Delta\right) \Phi$ is negative in the domain wall, where the magnetization is reduced.

The areal energy density of the domain wall is obtained by integrating the energy density over $x$, where corrections to $m(x)$ of order $1 / L$ are again relevant,

$$
\begin{aligned}
\Delta \sigma= & \int_{-\infty}^{\infty} d x\left[-\frac{c d}{2\left(d+a / 2 r_{0}^{2}\right)} \Delta m^{2}\right. \\
& \left.-\frac{1}{4}\left(d-\frac{a}{2 r_{0}^{2}}\right)\left(\Delta m^{2}\right)^{2}+\frac{a}{8}\left(\partial_{x} \Delta m^{2}\right)^{2}\right],
\end{aligned}
$$

with $\Delta m^{2} \equiv m^{2}( \pm \infty)-m^{2}(x)$. The dependence of $\Delta \sigma$ on the coupling $a \propto \eta^{2}$ is shown in the inset in Fig. 4. $\Delta \sigma$ first decreases with increasing coupling and then increases again, finally diverging as $d^{\prime}=d-a / 2 r_{0}^{2}$ goes to zero.
For larger $\left|c r_{0}^{2} / b\right|$, the divergence is not reached, since the condition $m_{0}=m_{\text {sing }}$ (i.e., $T=T^{*}$ ) is satisfied first. The initial decrease is dominated by the $1 / L$ term in $\Delta m^{2}(x)$ far from the wall, i.e., from the redistribution of carriers. In this regime, domain walls are (slightly) less costly than they would be without coupling. The strong increase comes mostly from the increased width due to Coulomb repulsion. Domain walls could be observed in the real-space experiments discussed above. The charged layer should also affect electronic transport through domain walls.

Conclusions. - The carrier-concentration dependence of the magnetization in DMS introduces a characteristic temperature $T^{*}<T_{c}$ such that the mean-field magnetization $m$ and excess carrier density $\delta n$ show periodic modulations for $T<T^{*}$, whereas $m$ is homogeneous and $\delta n=0$ above $T^{*} . T_{c}-T^{*}$ can be of the order of $10 \mathrm{~K}$ in $p$-type DMS. The modulation is strongly anharmonic, and amplitude and wavelength increase for decreasing temperature, starting from zero at $T^{*}$. For $T \geq T^{*}$, the equilibrium state is homogeneous, but the coupling between magnetism and carrier concentration leads to the appearance of a negatively charged layer in the vicinity of a domain wall for $p$-type DMS.

*Electronic address: ctimm@ku.edu

[1] S. A. Wolff et al., Science 294, 1488 (2001).

[2] I. Žutić, J. Fabian, and S. Das Sarma, Rev. Mod. Phys. 76, 323 (2004).

[3] H. Ohno, Science 281, 951 (1998); J. Magn. Magn. Mater. 200, 110 (1999); T. Dietl, Semicond. Sci. Technol. 17, 377 (2002).

[4] C. Timm, J. Phys. Condens. Matter 15, R1865 (2003).

[5] H. Ohno et al., Nature (London) 408, 944 (2000).

[6] H. Boukari et al., Phys. Rev. Lett. 88, 207204 (2002).

[7] K. W. Edmonds et al., Appl. Phys. Lett. 81, 3010 (2002).

[8] A. M. Nazmul et al., Jpn. J. Appl. Phys. 43, L233 (2004).

[9] X. Liu et al., Phys. Rev. B 71, 035307 (2005).

[10] H. Kato et al., Jpn. J. Appl. Phys. 44, L816 (2005).

[11] M. B. Salamon and M. Jaime, Rev. Mod. Phys. 73, 583 (2001).

[12] C. H. Chen, S.-W. Cheong, and A. S. Cooper, Phys. Rev. Lett. 71, 2461 (1993).

[13] J. H. Cho, F. C. Chou, and D. C. Johnston, Phys. Rev. Lett. 70, 222 (1993); J. M. Tranquada et al., Nature (London) 375, 561 (1995).

[14] T. Dietl et al., Science 287, 1019 (2000).

[15] C. Timm, F. Schäfer, and F. von Oppen, Phys. Rev. Lett. 89, 137201 (2002).

[16] T. Shono et al., Appl. Phys. Lett. 77, 1363 (2000).

[17] U. Welp et al., Phys. Rev. Lett. 90, 167206 (2003).

[18] T. Jungwirth et al., Phys. Rev. B 66, 012402 (2002).

[19] If one imposes the same boundary conditions for $T<T^{*}$, the solution must cross the singularities at $\pm m_{\text {sing. Only an }}$ oscillatory solution of type 1 is possible. 